\title{
Novel-agent combination therapies in chronic lymphocytic leukemia: the law of relative contributions
}

\author{
Anthony R. Mato and Lindsey E. Roeker \\ Memorial Sloan Kettering Cancer Center, New York, NY, USA \\ E-mail: Matoa@mskcc.org \\ doi:10.3324/haematol.2021.280217
}

In this issue of Haematologica, Moreno et $a l^{1}{ }^{1}$ present the final analysis of the randomized, phase III iLLUMINATE trial. In this study, the combination of the Bruton tyrosine kinase (BTK) inhibitor ibrutinib (administered in a treatto-progression, continuous fashion) plus the anti-CD20 monoclonal antibody obinutuzumab was compared to time-limited chlorambucil plus obinutuzumab in the front-line setting. Now with a median of 45 months of follow-up, the data confirm an impressive progression-free survival (PFS) advantage of ibrutinib plus obinutuzumab over chlorambucil plus obinutuzumab (median PFS not reached for ibrutinib plus obinutuzumab vs. 22 months for chlorambucil plus obinutuzumab, hazard ratio $=0.25$, 95\% confidence interval: 0.16-0.39, $P<0.0001)$ without a clear advantage in overall survival (hazard ratio $=1.08$, $P=0.793$ ), although it should be noted that treatment crossover was allowed. For ibrutinib plus obinutuzumab, the overall response rate, complete response rate and rate of undetectable minimal residual disease were also impressive at 91\%, 42\% and 38\%, respectively. The iLLUMINATE trial also allowed enrollment of patients with chromosome 17p deletion [(del17p)] providing efficacy data for ibrutinib-based therapy in this high-risk population of patients.

Taken in the context of the current treatment landscape, these data from iLLUMINATE should trigger important considerations regarding the relative contributions of drugs. While the ibrutinib plus obinutuzumab combination is unequivocally superior to the chlorambucil-based control arm in terms of PFS, we do not gain insight into how obinutuzumab adds to the already herculean activity of ibrutinib in the front-line setting as this trial did not include an ibrutinib monotherapy arm. In the current pandemic, assessing the contribution of obinutuzumab is particularly relevant given that the addition of an antiCD20 monoclonal antibody will blunt or eliminate humoral responses to SARS-Cov-2 mRNA vaccines (all risk without a proven reward). Additionally, lessons learned from recent randomized trials in chronic lymphocytic leukemia (CLL) remind us that only with proper assessment of relative contributions can one determine the true risk versus benefit of a combination. For example, two prior randomized clinical trials compared ibrutinib to ibrutinib with the anti-CD20 monoclonal antibody rituximab and did not demonstrate a PFS or overall survival advantage for ibrutinib plus rituximab over ibrutinib alone. ${ }^{2,3}$ Based on these trials which provided clarity on the relative contribution of rituximab to ibrutinib, nearly all clinicians favor ibrutinib monotherapy over ibrutinib plus rituximab combination therapy. In the ELEVATE-TN study the second generation BTK inhibitor acalabrutinib was compared alone and in combination with obinutuzumab to chlorambucil plus obinutuzumab in the front-line setting. ${ }^{4}$ In this three-arm, randomized, phase III, clinical trial, Sharman et al. demonstrated a PFS advantage for the combination of acalabrutinib plus obinutuzumab over acalabrutinib monotherapy. The ELEVATE-TN study design should be celebrated, as it allowed clinicians to examine the benefit of adding obinutuzumab to acalabrutinib and supports the use of the novel agent-based combination acalabrutinib plus obinutuzumab. Given differences in patient populations, clinical trial designs, and biases inherent to crosstrial comparisons of different agents, data from ELEVATE TN should not be freely extrapolated to other BTK inhibitors plus obinutuzumab combinations. This body of literature catalyzes careful consideration of how to design future novel agent combination studies in CLL to adequately assess the relative safety and efficacy contributions of individual components of a multi-agent combination regimen.

As future efforts to optimize treatment of CLL focus on novel agent-based doublet and triplet therapy, we must consider the relative contributions of each component. Phase I/II studies examining combination therapies have demonstrated safety and efficacy, leading to the development of phase III studies exploring the combinations. Rational phase III study design requires consideration of whether the combination is absolutely required to achieve the desired clinical outcome. For example, in the recently reported GLOW trial, the combination of ibrutinib and venetoclax demonstrated superior PFS to the chlorambucil plus obinutuzumab regimen. ${ }^{5}$ While the GLOW study is undoubtedly positive with regard to its primary PFS endpoint, the trial does not allow us to determine the relative contributions of the components of the ibrutinib plus venetoclax combination to safety or efficacy. With the current data, we cannot determine whether the combination is better than monotherapy or a single novel agent in combination with obinutuzumab. Novel agent monotherapy control arms are noticeably absent in many of the currently accruing trials examining novel agent-based doublet and triplet therapies, so assessment of the 
relative contribution of each component will not be possible. A major unanswered question in the treatment of CLL is which patients require monotherapy, which require doublet therapy and which require triplet therapy. ${ }^{6}$ Active phase III studies, with the notable exception of CLL17 (NCT04608318), are not designed to answer this question. The issue of relative contribution is ubiquitous in oncology, as recently highlighted in an excellent review by Brewer et al., which focused on regulatory considerations for the contribution of effects of drugs used in combination regimens. ${ }^{7}$ The authors concluded that for the approval of combination regimens, "it is necessary to demonstrate the contribution of effect of each monotherapy to the overall combination." These authors further assessed the strengths and weaknesses of various methods we can use to assess relative contribution of effect. Perhaps the most applicable solution to the issue at hand in CLL is a multi-arm adaptive trial design in which patients can be randomized to trial arms performing at a "prespecified level of efficacy." Such an approach can yield data on the contribution of novel agent monotherapy control arms with the ability to drop such controls if they are underperforming. This adaptive approach was employed in UNITY-CLL, a front-line clinical trial in CLL which successfully assessed the relative contributions of umbralisib and ublituximab monotherapies to the combination of umbralisib and ublituximab. ${ }^{8}$

In their article, Moreno et al. ${ }^{1}$ eloquently discuss the issue of relative contribution. Most notably, the authors com- pare the 48-month PFS of ibrutinib monotherapy from the RESONATE- 2 trial to the 48-month PFS of the ibrutinib plus obinutuzumab iLLUMINATE regimen demonstrating nearly identical PFS ( 75\%) at that time point. As we aim to understand the role of novel agent doublet and triplet therapy in CLL, iLLUMINATE shines a light on an ever-important issue to be considered in the next generation of clinical trials: the law of relative contributions.

\section{Disclosures}

ARM has received research support from TG Therapeutics, Pharmacyclics, Abbvie, Adaptive Biotechnologies, Johnson and Johnson, Acerta / AstraZeneca, DTRM BioPharma, Sunesis, BeiGene, Genentech, Genmab, Janssen and Loxo Oncology, Nurix; and has provided advisory, consultancy or data safety and monitoring board services for TG Therapeutics, Pharmacyclics, Adaptive Biotechnologies, Abbvie, Johnson and Johnson, Acerta / AstraZeneca, DTRM BioPharma, Sunesis, AstraZeneca, BeiGene, Genentech, Janssen and Loxo Oncology. LER has received research support from Pfizer, Loxo Oncology, and Aptose Biosciences; has served as a consultant for AbbVie, AstraZeneca, Beigene, Janssen, Loxo Oncology, Pharmacyclics, Pfizer, TG Therapeutics, and Vaniam group; and holds minority ownership interest in Abbott Laboratories.

\section{Contributions}

Both authors contributed equally.

\section{References}

1. Moreno C, Greil R, Demirkan F, et al. First-line treatment of chronic lymphocytic leukemia with ibrutinib plus obinutuzumab versus chlorambucil plus obinutuzumab: final analysis of the randomized, phase III iLLUMINATE trial. Haematologica. 2022;107(9):2108-2120.

2. Woyach JA, Ruppert AS, Heerema NA, et al. Ibrutinib regimens versus chemoimmunotherapy in older patients with untreated CLL. N Engl J Med. 2018;379(26):2517-2528.

3. Burger JA, Sivina M, Jain N, et al. Randomized trial of ibrutinib vs ibrutinib plus rituximab in patients with chronic lymphocytic leukemia. Blood. 2019;133(10):1011-1019.

4. Sharman JP, Egyed M, Jurczak W, et al. Acalabrutinib with or without obinutuzumab versus chlorambucil and obinutuzmab for treatment-naive chronic lymphocytic leukaemia (ELEVATE TN): a randomised, controlled, phase 3 trial. Lancet. 2020;395(10232):1278-1291.

5. Kater A, Owen C, Moreno C, et al. Fixed duration ibrutinib and venetoclax $(I+V)$ versus chlorambucil plus obinutuzumab (CLB+O) for first-line (1L) chronic lymphocytic leukemia (CLL): primary analysis of the phase 3 GLOW study. HemaSphere. 2021;5(S2):LB1902.

6. Sarraf Yazdy M, Mato AR, Cheson BD. Combinations or sequences of targeted agents in CLL: is the whole greater than the sum of its parts (Aristotle, $360 \mathrm{BC}$ )? Blood. 2019;133(2):121-129.

7. Brewer JR, Chang E, Agrawal S, et al. Regulatory considerations for contribution of effect of drugs used in combination regimens: renal cell cancer case studies. Clin Cancer Res. 2020;26(24):64066411.

8. Gribben JG, Jurczak W, Jacobs RW, et al. Umbralisib plus ublituximab (U2) is superior to obinutuzumab plus chlorambucil $(\mathrm{O}+\mathrm{Chl})$ in patients with treatment naïve $(\mathrm{TN})$ and relapsed/refractory $(R / R)$ chronic lymphocytic leukemia (CLL): results from the phase 3 Unity-CLL study. Blood. 2020;136:37-39. 